\title{
Electromagnetic Optimization of Passive RFID Sensor Nodes
}

\author{
Cecilia Occhiuzzi \\ DISP, University of Roma Tor Vergata, \\ Via del Politecnico, 1 \\ 00133, Roma (ITALY) \\ Email: occhiuzzi@disp.uniroma2.it
}

\author{
Gaetano Marrocco \\ DISP, University of Roma Tor Vergata, \\ Via del Politecnico, 1 \\ 00133, Roma (ITALY) \\ Email: marrocco@disp.uniroma2.it
}

\begin{abstract}
RFID passive tags are nowadays starting to be considered more than labeling devices: by properly analyzing the two-ways communication link it is possible to get information about the state of a tagged object, without any specific embedded sensor or local power supply. Despite of the generality and the straightforwardness of the approach, the design of such a class of devices requires specific strategies to make the radio-sensors able to properly track the evolution of the phenomena under observation, jointly optimizing communication and sensing requirements. In this paper the optimization problem is formalized by means of convenient matching charts and evaluated in realistic experimental examples.
\end{abstract}

\section{INTRODUCTION}

The possibility to use passive tags as sensing devices of objects, people and environment is nowadays one of the most interesting and promising application of passive UHF Radio Frequency Identification (RFID) technology. Many different employments have been already envisaged and experimented, demonstrating the feasibility of the idea but, at the same time, the limitation of the actual design methodologies [1].

The rationale of the tag-as-sensor is based on the fact that, since the tag's input impedance and gain depend on the surrounding environment, any of its physical or chemical variation could affect the tag's performance and be remotely detected by the reader. The generality of this basic principle allows in theory any passive tag to be used as a sensor of "effective" permittivity changes. However, this class of devices should be able to properly track the evolution of the phenomena under observation, being for example monotonic, single valued and sensitive enough at least in the most critical ranges. Communication and sensing features demand for opposite requirements, since the sensitivity of the tag-as-sensor to the variation of the process under observation is usually paid in terms of a worsening of the power scavenging capabilities and in particular of the read distance.

Since there is no decoupling from the operative and structural point of view between antenna and sensor, the traditional design procedures have to be revisited or even rethought in order to handle at the same time both sensing and communication needs. In this paper the optimization problem is formalized in terms of the shaping of the tag's response to the change of the observed process by means of convenient matching charts, useful to suggest the most appropriate choice of the antenna's impedance. The method is evaluated by an example of object with time-varying permittivity.

\section{METHOD}

Let $\Psi(t)$ denote a local physical, chemical or geometrical parameter of the environment surrounding the tag which has to be monitored by the RFID platform. The RFID communication exploits a two-way link: a direct one, wherein the key-issue is the scavenging of power at the chip's port so that the tag may activate and perform actions, and a reverse link wherein the tag transmits its data to the reader by changing its reflectivity through impedance modulation. The link [2] for UHF-RFID systems (overall World-wide band: 866-956 MHz) is commonly characterized under the far field assumption by using the Friis formula for the direct path (1), and the radar cross-section for the reverse one (2):

$$
\begin{gathered}
P_{R \rightarrow T}[\Psi]=\left(\frac{\lambda_{0}}{4 \pi d}\right)^{2} P_{i n} G_{R}(\theta, \phi) G_{T}[\theta, \phi, \Psi(t)] \tau\left([\Psi(t)] \eta_{p}\right. \\
P_{R \leftarrow T}[\Psi]=\frac{1}{4 \pi}\left(\frac{\lambda_{0}}{4 \pi d^{2}}\right)^{2} P_{i n} G_{R}^{2}(\theta, \phi) \eta_{p}^{2} r c s_{T}[\theta, \phi, \Psi(t)]
\end{gathered}
$$

where $d$ is the reader-tag distance, $G_{R}(\theta, \phi)$ is the gain of the reader antenna, $G_{T}[\theta, \phi, \Psi(t)]$ is the gain of the tag's antenna. $P_{i n}$ is the power entering the reader's antenna, $\eta_{p}$ is the polarization mismatch between the reader and the tag, and $\tau[\Psi(t)]$ is the power transmission coefficient of the tag :

$$
\tau[\Psi]=\frac{4 R_{\text {chip }} R_{a}[\Psi]}{\left|Z_{\text {chip }}+Z_{a}[\Psi]\right|^{2}}
$$

with $Z_{\text {chip }}$ input impedance of the RFID integrated circuit (IC) and $Z_{a}$ input impedance of the antenna. $r c s_{T}$ is the tag's radar cross-section, related to the modulation impedance $Z_{\text {mod }}$ of the microchip to encode the low and high digital states:

$$
\operatorname{rcs}_{T}[\Psi]=\frac{\lambda_{0}^{2}}{4 \pi} G_{T}^{2}[\theta, \phi, \Psi(t)]\left(\frac{2 R_{a}[\Psi]}{\left|Z_{\text {mod }}+Z_{a}[\Psi]\right|}\right)^{2}
$$


The backscattered power $P_{R \leftarrow T}$ is directly measurable by the reader in terms of the Received Signal Strength Indicator (RSSI), here assumed to correspond [3] to the binary modulating state having $Z_{\text {mod }}=Z_{\text {chip }}$.

Finally, forward (1) and backward (2) powers may be combined at turn-on, e.g. when the power collected by the tag $P_{R \rightarrow T}(\Psi)$ equals the IC sensitivity $P_{c h i p}$, with the purpose to drop out the influence of the distance and of the reader's and tag's gains and orientation [4]. A non-dimensional indicator, denoted as Analog Identifier (AID), can be hence introduced:

$$
A I D[\Psi]=\frac{P_{\text {chip }}}{\sqrt{P_{R \leftarrow T}[\Psi] \cdot P_{\text {in }}^{t o}[\Psi]}}=2 R_{\text {chip }}\left|Z_{\text {chip }}+Z_{a}[\Psi]\right|
$$

and it is very useful when the interrogation set-up changes (position and orientation) in successive measurements. The AID, in fact, just depends on the impedance mismatch and is immune to the interrogation modalities.

Generally, the direct link imposes the bottleneck of the whole tag-reader communication and it limits the maximum read range. The parameter that represents the tag's scavenging capabilities is the realized gain $G_{\tau}=G_{T} \tau$, e.g the gain of the tag's antenna scaled by the mismatch to the RFID IC.

A trade-off solution to the sensing-communication problem is to observe the state of the object $\Psi(\mathrm{t})$ through a measurable quantity whose variation is not necessarily associated to a worsening of the communication performance. Such an indicator should be optimally a parameter of the reverse-link such as the backscattered power which is however orientationand position-dependent. If some degradation can be accepted, the Analog IDentifier (AID) is also a convenient metrics since combines both the direct and the reverse links to provide a sensing information independently on the reader-tag position and orientation as well as on the interaction with the surrounding environment.

Due to the small size of the UHF tag's antenna, there are very few degrees of freedom in shaping the gain. The direct and reverse links could be therefore analyzed in term of the only input impedance by jointly controlling the power transmission coefficient $\tau(\Psi)$ and the $A I D(\Psi)$, or even $\tau(\Psi)$ and $\sqrt{\operatorname{rcs}_{T}(\Psi)}$.

\section{A. $\{\tau, A I D\}$ Chart}

Power transmission coefficient and Analog Identifier could be usefully expressed in terms of normalized input impedance [5] $r_{a}=R_{A} / R_{\text {chip }}, x_{a}=X_{a} / X_{\text {chip }}$, with $Q=$ $\left|X_{\text {chip }}\right| / R_{\text {chip }}$ :

$$
\begin{gathered}
\tau[\Psi]=\frac{4 r_{a}}{\left|1+r_{a}+j Q\left(1+x_{a}\right)\right|^{2}} \\
A I D[\Psi]=\sqrt{\tau[\Psi] \frac{1}{r_{a}[\Psi]}}=\frac{2}{\left|1+r_{a}+j Q\left(1+x_{a}\right)\right|}
\end{gathered}
$$

Having fixed $Q$, a chart of $\{\tau$, AID $\}$ isolines can be produced by varying $r_{a}$ and $x_{a}$ so that the communication and

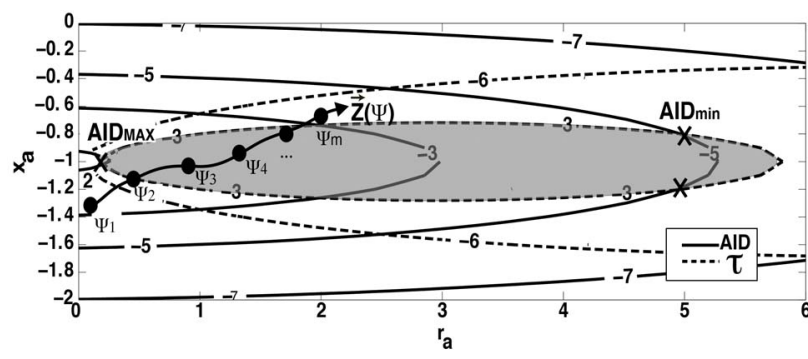

Figure 1. Iso-line of constant AID and $\tau$ on normalized antenna impedance plane. In grey the area corresponding to $\tau_{\min }=-3 d B$. The curve $\vec{Z}[\Psi]=$ $r_{a}(\Psi) \hat{i}+x_{a}(\Psi) \hat{j}$ indicates a possible variation of the antenna's impedance all along the process in evolution.

the sensing characteristics of the tag are graphically presented with a same diagram (Fig.1). The eccentricity of the ellipses depends on the quality factor $Q$ of the microchip. The point $(1,-1)$ corresponds to the perfect matching condition between antenna and IC, for which $A I D=\tau=0 d B$ : the state of the process $\Psi$, real or virtual, for which the radio sensor shows the best matching to the IC is hereafter denoted matched state, $\Psi_{m}: \tau\left(\Psi_{m}\right)=1$.

The $\tau$-AID diagram can be considered as a kind of Sensing Smith Chart, in which the role of the frequency is replaced by the process $\Psi$. Each process in evolution from an initial to a final state, involving a modification of input impedance, can be traced over such a plane by a sequence of couplets $\left(r_{a}\left(\Psi_{n}\right), x_{a}\left(\Psi_{n}\right)\right)$ describing an oriented curve $\vec{Z}[\Psi]=r_{a}(\Psi) \hat{i}+x_{a}(\Psi) \hat{j} \in \mathbb{R}^{2}$. According to the intercepted isolines, the radio sensor will show different sensing and communication performances all along the process.

The matched state $\Psi_{m}$ can be chosen at the end, at the beginning or in the middle of the process $\Psi$, such to produce different orientations of $\vec{Z}[\Psi]$. The amplitude of the sensing response is instead mainly determined by the impedance evolution: the wider is the range of the input impedance variation, the longer is the path of $\vec{Z}(\Psi)$. Similar considerations can be done about its direction. In the most general case $\vec{Z}(\Psi)$ has a certain slope $\alpha$ respect to the $r_{a}$-axis, if the process produces predominantly variation of real part, the sensor response tends to be parallel to the $x_{a}$ axis $\left(\alpha=0^{\circ}\right)$; instead if the process's variation applies on the antenna reactance, a near vertical $\vec{Z}(\Psi)$ curve will be obtained $\left(\alpha=90^{\circ}\right)$. The slope of such a curve with respect to the $r_{a}$-axis plays an important role on the overall tag's sensitivity to the process evolution. Finally, since the AID isolines are monotonically distributed on the $\left(r_{a}, x_{a}\right)$ plane, the sensing response of the radio-sensor can be considered monotonic as well if $\vec{Z}[\Psi]$ doesn't show loops or twist.

To preserve a stable read-range of the tag during the whole evolution of the process, it is required that $\tau[\Psi]>\tau_{\text {min }}$. For instance the choice $\tau_{\min }=-3 d B$ will permit not to degrade the read range below the $70 \%$ of its maximum value corresponding for example to the initial (or final) state of the process. The vector $\vec{Z}\left(r_{a}, x_{a}\right)$ is hence forced to belong to 


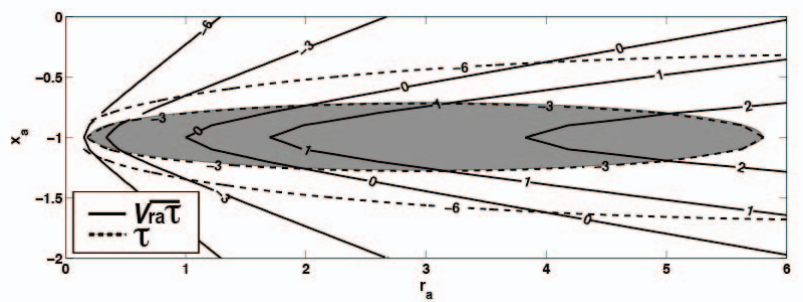

Figure 2. Iso-line of constant $r c s_{T}$ and $\tau$ on normalized antenna impedance plane. In grey the area corresponding to $\tau_{\min }=-3 d B$.

the shadowed elliptical region in Fig.1. The constraint on $\tau$ accordingly fixes the maximum variation of AID, in the specific: $-5 d B<A I D[\Psi]<2 d B$ as observed by the intersections between the isocurves. In general, for a real antenna, the path $\vec{Z}\left(r_{a}, x_{a}\right)$ can totally, partially or even not belong to such an area: only the dynamics of $\Psi$ inside the shadowed ellipse will be observed by the radio sensor.

The design procedures is hence aimed to confine $\vec{Z}\left(r_{a}, x_{a}\right)$ inside the $\tau[\Psi]>\tau_{\min }$ region. At this purpose, an antenna adapter $A A$ (e.g. a T-match structure or a lumped network [6]) can be included in the tag design to yield a transformed impedance $Z_{T}=A A \circ Z_{A}$ suitable to get the best response profile $\vec{Z}$ all along the process. The $A A$ can be considered as a filter with geometrical or electrical parameters to be found by any kind of optimization method.

\section{B. $\left\{\tau, \sqrt{r c s_{T}}\right\}$ Chart}

A similar sensing chart can be drawn by observing both direct and reverse link through the realized gain. To this purpose, it is convenient to express also the $\operatorname{rcs}_{T}(\Psi)$ in term of normalized input impedance:

$$
\operatorname{rcs}_{T}[\Psi]=\frac{\lambda_{0}^{2}}{4 \pi} G_{T}^{2}[\theta, \phi, \Psi] r_{a}[\Psi] \tau[\Psi]
$$

In order to drop out the influence of gain from both indicators it is better to consider the $\sqrt{r c s_{T}}$, such to have a chart of $\left\{\tau, \sqrt{r_{a} \tau}\right\}$ isolines (Fig.2). Similarly to the $\{$ AID,$\tau\}$ chart, also in this case it is possible to define a constraint on $\tau_{\text {min }}$ to preserve a stable read-range all along the process; e.g for $\tau_{\min }=-3 d B$ (gray ellipses in Fig.2) the maximum detectable sensing variation will be $-5 d B<\sqrt{r c s_{T}}<2 d B$.

It is interesting to notice, by comparing Fig.1 and Fig.2, that to maximize the sensing link is necessary to place $\vec{Z}[\Psi]$ approximately in the same manner on the plane $\left(r_{a}, x_{a}\right)$ : by designing a tag in such an area it is thus possible to optimize the variation of both analog identifier and backscattered power without extreme degradation of the communication performances.

The issue of maximizing the power transmission coefficient and the $\operatorname{rcs}_{T}$ in the design phase has been recently discussed in [7] by considering only the communication functionality of the tag: thanks to $\left\{\sqrt{r_{a} \tau}, \tau\right\}$ chart, instead, it is here demonstrated that, although it is not possible to contemporarily maximize for a same tag the power scavenging, i.e $\tau$, and the backscattered

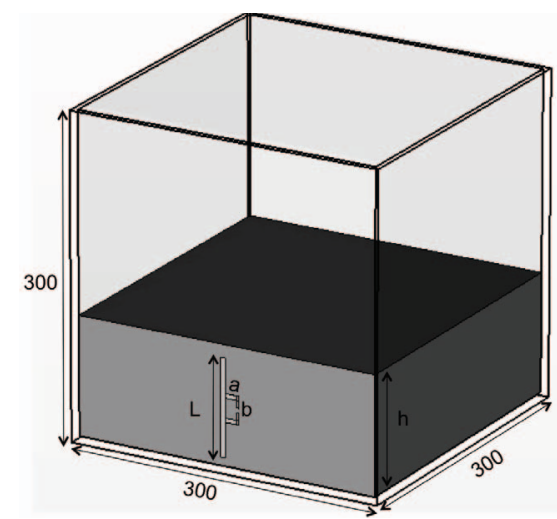

Figure 3. Passive T-matched tag to sense the level of sugar powder within a perspex box. Size after optimization in $\mathrm{mm}$ are $L=90, a=13, b=20$.

power, i.e $r c s_{T}$, it is however possible to dynamically optimize their coexistence for sensing purposes.

\section{A NumERICAL EXAMPLE}

It is now designed an optimized tag for the wireless monitoring of the filling level $(\Psi=h)$ of a perspex box containing sugar (FDTD model in Fig.3) [8]. The first guess for the tag-as-sensor is a simple half-wave dipole @870MHz. The considered RFID IC has impedance $Z_{I C}=17-j 190 \Omega$ and the corresponding $A I D-\tau$ chart is shown in Fig. 4(top). The communication performances are preserved by enforcing the constrain $\tau_{\min }=-1 d B$ (less than $10 \%$ reduction in the read range). Accordingly, the maximum variation of the AID could be at most $\triangle A I D=1-(-2 d B)=3 d B$ (Fig.4 top), while $\Delta \sqrt{r c s_{T}}=-2-(1.5 d B)=3.5 d B$ (Fig.4 middle). The response, say $\vec{Z}_{A}[\Psi]$, of the monopole lies outside the useful area, it exhibits a non monotonic profile and hence it is not suited to sensing and neither to communication. An impedance adapter is thus added to reshape the curve (Fig.3). The form factor $(a, b)$ of the T-match is optimized by means of a Genetic Algorithm with the aim to move the tag's response (now denoted as $\vec{Z}_{B}$ in Fig.4 top) inside the shadowed region $\tau>-1 d B$ and to achieve a monotonic profile. In this case, $\vec{Z}_{B}$ has been anchored to the point (1-1) by choosing a matched state $\Psi_{m}=2 \mathrm{~cm}$.

Fig. 4 bottom shows the corresponding AID , $\sqrt{r c s}$ and $\tau$ parameters versus the sugar's height for the optimized tag with $a=13 \mathrm{~mm}, b=20 \mathrm{~mm}$. As required the power transfer coefficient is nearly unchanged around the matching condition which means that the reader will be able to monitor the level of the sugar with $0.3 \mathrm{~dB} / \mathrm{cm}$ sensitivity of the AID and $\sqrt{r c s_{T}}$ and with negligible variation of the read range.

\section{CONCLUSION}

The design of RFID radio sensors can be optimized by jointly considering communication and sensing features. The optimization can be guided by using innovative nomograms able to represent the key parameters of the direct link, mainly 

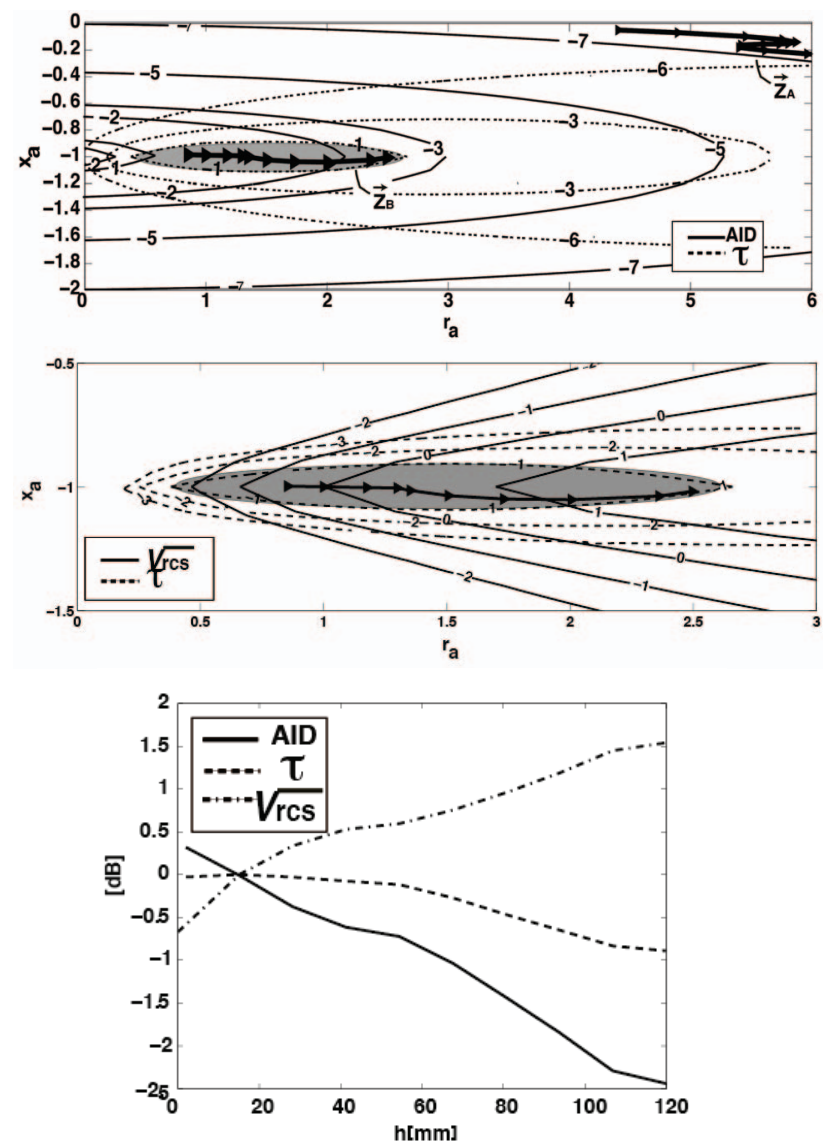

Figure 4. Top) $A I D-\tau$ chart. Middle) $\sqrt{r c s_{T}}-\tau$ chart. In grey the area corresponding to $\tau_{\min }=-1 d B . \vec{Z}_{A}$ is the response curve of the dipole, transformed by the T-match in $\vec{Z}_{B}$. Bottom) $A I D, \tau$ and $\sqrt{r c s_{T}}$ profile versus the filling level $h[\mathrm{~mm}]$.

devoted to the communication, and the reverse link, mainly focused instead on the sensing activity.

It is finally worth noticing the flexibility of the proposed procedure which permits to adapt a same shape of tag to different processes in evolution by just changing the shape factor of the antenna adapter, such to confine confine $\vec{Z}\left(r_{a}, x_{a}\right)$ inside the $\tau[\Psi]>\tau_{\min }$ region in the most suitable way.

\section{ACKNOWLEDGMENT}

Work developed under project PRIN-2008: MULTI-TAG funded by Italian Ministry of University.

\section{REFERENCES}

[1] G. Marrocco, "Pervasive electromagnetics: sensing paradigms by passive rfid technology," Wireless Communications, IEEE, vol. 17, pp. $10-17$, december 2010 .

[2] P. Nikitin and K. Rao, "Theory and measurement of backscattering from rfid tags," Antennas and Propagation Magazine, IEEE, vol. 48, pp. 212 -218 , dec. 2006

[3] F. Fuschini, C. Piersanti, F. Paolazzi, and G. Falciasecca, "Analytical approach to the backscattering from uhf rfid transponder," Antennas and Wireless Propagation Letters, IEEE, vol. 7, pp. 33 -35, 2008.
[4] G. Marrocco, "Rfid grids: Part i:electromagnetic theory," Antennas and Propagation, IEEE Transactions on, vol. 59, pp. 1019 -1026, march 2011

[5] K. Rao, P. Nikitin, and S. Lam, "Impedance matching concepts in rfid transponder design," in Automatic Identification Advanced Technologies, 2005. Fourth IEEE Workshop on, pp. 39 - 42, oct. 2005.

[6] G. Occhiuzzi, C. ; Marrocco, "Human body sensing: A pervasive approach by implanted rfid tags," in Applied Sciences in Biomedical and Communication Technologies (ISABEL), 2010 3rd International Symposium on, 2010.

[7] J. Bolomey, S. Capdevila, L. Jofre, and J. Romeu, "Electromagnetic modeling of rfid-modulated scattering mechanism. application to tag performance evaluation," Proceedings of the IEEE, vol. 98, pp. $1555-$ 1569 , sept. 2010.

[8] G. Marrocco and F. Amato, "Self-sensing passive rfid: From theory to tag design and experimentation," in Microwave Conference, 2009. EuMC 2009. European, pp. 001 -004, 29 2009-oct. 12009. 